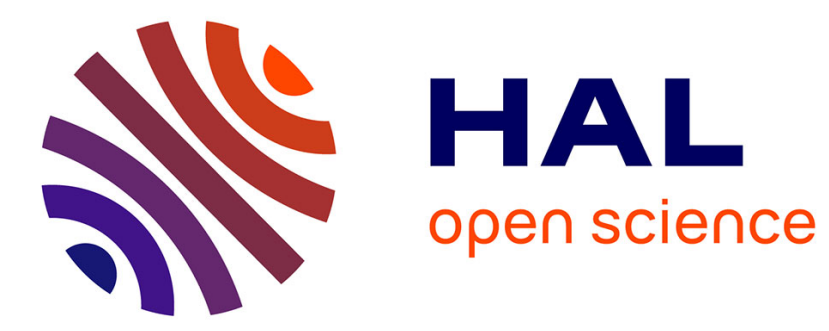

\title{
Emergence of humanoid walking behaviors from mixed-integer model predictive control
}

\author{
Aurelien Ibanez, Philippe Bidaud, Vincent Padois
}

\section{To cite this version:}

Aurelien Ibanez, Philippe Bidaud, Vincent Padois. Emergence of humanoid walking behaviors from mixed-integer model predictive control. IEEE/RSJ International Conference on Intelligent Robots and Systems, Sep 2014, Hong-Kong, China. pp.4014 - 4021, 10.1109/IROS.2014.6943127 . hal-01116331

\section{HAL Id: hal-01116331 \\ https://hal.science/hal-01116331}

Submitted on 13 Feb 2015

HAL is a multi-disciplinary open access archive for the deposit and dissemination of scientific research documents, whether they are published or not. The documents may come from teaching and research institutions in France or abroad, or from public or private research centers.
L'archive ouverte pluridisciplinaire HAL, est destinée au dépôt et à la diffusion de documents scientifiques de niveau recherche, publiés ou non, émanant des établissements d'enseignement et de recherche français ou étrangers, des laboratoires publics ou privés. 


\title{
Emergence of humanoid walking behaviors from Mixed-Integer Model Predictive Control
}

\author{
Aurelien Ibanez ${ }^{1}$, Philippe Bidaud ${ }^{1,2}$ and Vincent Padois ${ }^{1}$
}

\begin{abstract}
Balance strategies range from continuous postural adjustments to discrete changes in contacts: their simultaneous execution is required to maintain postural stability while considering the engaged walking activity. In order to compute optimal time, duration and position of footsteps along with the center of mass trajectory of a humanoid, a novel mixed-integer model of the system is presented. The introduction of this model in a predictive control problem brings the definition of a MixedInteger Quadratic Program, subject to linear constraints. Simulation results demonstrate the simultaneous adaptation of the gait pattern and posture of the humanoid, in a walking activity under large disturbances, to efficiently compromise between task performance and balance. In addition, a push recovery scenario displays how, using a single balance-performance ratio, distinct behaviors of the humanoid can be specified.
\end{abstract}

\section{INTRODUCTION}

Humanoid robots employ locomotion systems which are essentially of hybrid nature, combining discrete supports and multi-body dynamics. Legged locomotion couples these two sub-systems in different ways, and distinct behaviors emerge depending on the desired performance and constraints [1].

The choice of balance strategy is all the more important in situations where the robot undergoes large physical disturbances. Appropriate strategies in such cases generally lies between whole-body balancing and changes in the base of support (BoS). The robotics literature presents various solutions in this range, involving torque compensation [2], bracing behaviors [3] at the center of mass (CoM), angular momentum rejection [4] and shifts in foot placement [4], [5].

Changes in the BoS, however, have the potential to provide a greater degree of stabilization than whole-body adjustments in a fixed-support configuration [6], and may, on one hand, even be necessary depending on the activity and the constraints the system is subject to. On the other hand control of these changes may be more challenging. It requires indeed the determination of the adequate time, duration, distance and direction of the shift, while regarding both constraints on the system and the desired motion of the robot. With the Capture Region approach [4], Pratt et al. propose to solve the problem of when and where to take a step, along with suitable CoM and angular momentum behaviors. Although it has largely demonstrated its efficiency in push-recovery

\footnotetext{
${ }^{1}$ Aurelien Ibanez, Philippe Bidaud and Vincent Padois are with:

- Sorbonne Universités, UPMC Univ Paris 06, UMR 7222, Institut des Systèmes Intelligents et de Robotique, F-75005, Paris, France

- CNRS, UMR 7222, Institut des Systèmes Intelligents et de Robotique, F-75005, Paris, France \{ibanez, bidaud, padois\}@isir.upme.fr

${ }^{2}$ Philippe Bidaud is with the ONERA, 91123 Palaiseau, France philippe.bidaudeonera.fr
}

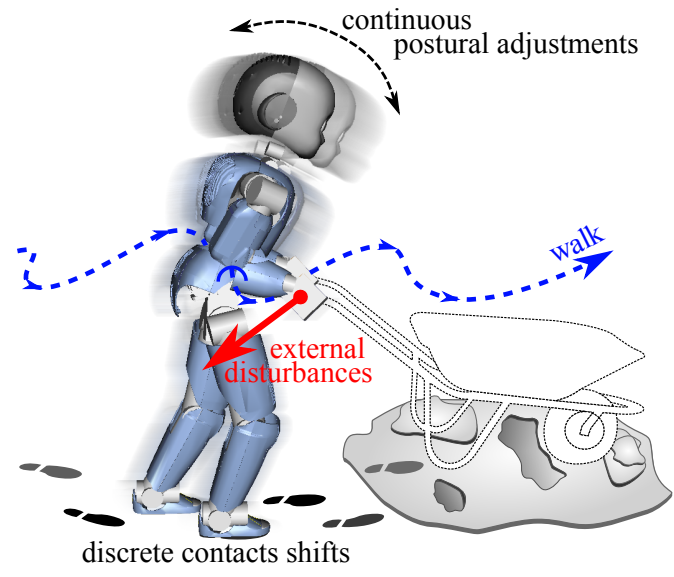

Fig. 1. Illustration of the hybrid nature of safe biped walking: the walking task induces discrete changes in the base of support, while balance is maintained through continuous postural adjustments and adaptation of the contacts position.

cases, this method lacks consideration of the constraints the robot is subject to and of the engaged activity, which could potentially affect the feasibility of the expected step. Furthermore, little insight on the suitable duration of the step is given.

Predictive approaches are appropriate to preview the influence of the duration and placement of the step taken, and can be applied to the Capture Region method as Krause et al. [7] propose in a Model Predictive Control (MPC) framework. MPC indeed provides a future time window to estimate the evolution of the system's state, and the formulation of an optimization problem is favorable to the consideration of constraints and objectives the robot must comply with. However, the additional complexity generally requires the use of reduced models, constraints approximations and predefined entities or heuristics in order to obtain computationally-efficient formulations. Moreover the hybrid nature of biped walking, involving continuous evolution of the system's motion and discrete changes in constraints and forces acting on it as illustrated in Figure 1, tends to prevent straightforward formulations. In order to confine the resolution to the continuous, smooth part of the problem, Herdt et al. [5] propose to exploit a previously given horizon of contacts activation to simultaneously optimize the CoM trajectory with contacts positions. Another method consists in regularizing the problem: Mordatch et al. [8] make for example use of hard-constraints smoothing for rigid contacts, in order to ensure regularity of the model. 
This papers proposes an original approach to the capture, in a predictive framework, of the influence of both CoM dynamics and changes in the BoS with respect to constraints on the system and the ongoing walking activity. No regularization of the hybrid nature of biped walking is carried out, and discrete events are described using a computationally favorable, redundant set of highlyconstrained integer variables. Behavior of the system is specified at a high level, as a sole ratio between balance and performance of the walking activity. The resulting MPC problem takes the form of a quadratic, linearly constrained mixed-integer program (MIQP) which allows to determine, over a preview horizon, an optimal strategy between changes in the $\mathrm{BoS}$ and $\mathrm{CoM}$ behavior, subject to multiple constraints, maximizing balance and performance of a walking activity.

This work is presented as follows. A linearly-constrained, mixed-integer set of variables is first introduced in Section II to characterize admissible changes in the BoS. The ZeroMoment Point (ZMP) approach is employed to capture, in terms of balance, the state of the system with respect to the BoS. The resulting model allows the linear expression of several constraints related to the walking activity.

Section III employs this model of the system in an MPC framework, and defines the control objective as a compromise between balance and performance of the walking task, which is formulated as the tracking of a desired CoM trajectory. An optimal horizon of changes in the BoS and CoM trajectory is computed from a MIQP, without the use of pre-defined gait patterns or heuristics.

Simulations results in Section IV validates this approach in various scenarii. Performance of the tracking task is demonstrated in a sinusoidal trajectory tracking case, and the introduction of large physical disturbances on the robot exhibits the conjoint adaptation of gait pattern and CoM trajectory. Last, the influence of the balance and walking weights is exhibited in a push recovery case where the system behavior, as a response to an unknown external action, significantly varies for different values of the weights ratio.

\section{ZERO-MOMENT POINT, MIXED-INTEGER BIPED MODEL}

In order to adopt the appropriate balance strategy, control algorithms should exploit a model of the system describing the effect of various quantities of interest on the postural stability of the robot. Indeed, employing this model to evaluate the performance of different evolutions of each of these quantities may lead to the identification of the best combination to select.

Under several hypotheses, the Zero-Moment Point [9] approach captures the balance state of the system by relating its CoM dynamics to the base of support. However, the hybrid nature of biped walking clearly differentiates CoM dynamics changes from BoS shifts: the former is continuous in essence, while the latter is restrained to discrete events.
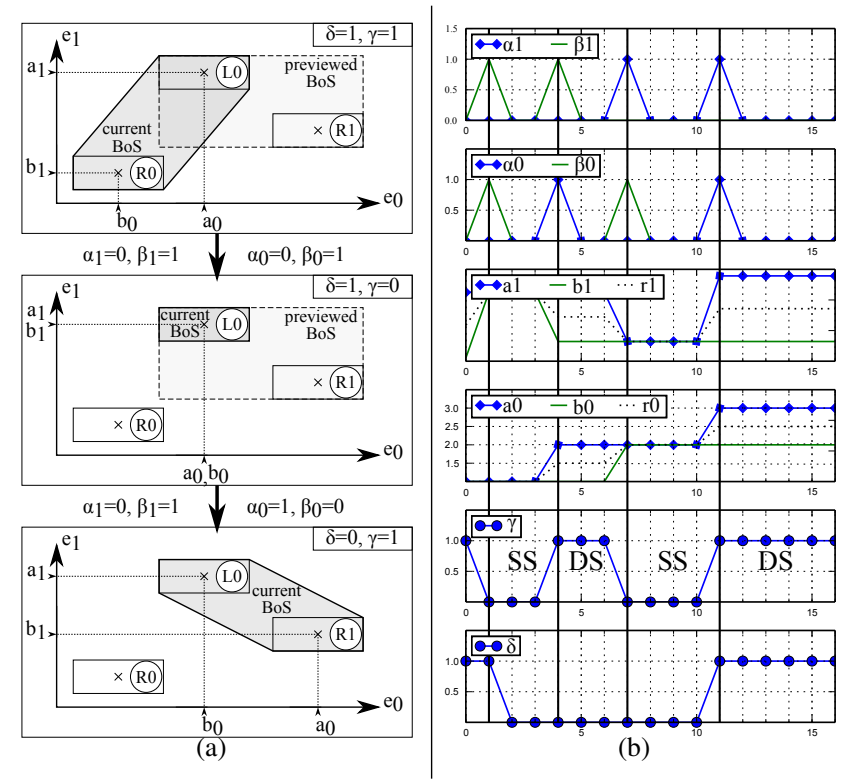

Fig. 2. Mixed-integer contact state description -2(a): Real valued $\boldsymbol{a}$ and $\boldsymbol{b}$ describe bounds of the position of the feet in contact, with their respective binary rising/falling edges $\boldsymbol{\alpha}$ and $\boldsymbol{b}$. Binary variable $\gamma$ differentiates single and double support phases, and $\delta$ restrains the evolution of bounds $\boldsymbol{a}, \boldsymbol{b}$ during transitions from double to single support. Current BoS is linearly expressed (dark gray) and previewed BoSs are approximated by their bounding box (light gray). -2(b): typical evolution of linearly-constrained variables $(\boldsymbol{a}, \boldsymbol{b}, \boldsymbol{\alpha}, \boldsymbol{\beta}, \delta, \gamma)$, related to the base of support. Variable $\boldsymbol{r}=(\boldsymbol{a}+\boldsymbol{b}) / 2$ is displayed here to describe the center of the supporting surface.

MPC methods, writing the control problem as an optimization problem, generally consider the continuous members of the ZMP model solely as degrees of freedom (DoF). Kajita et al. [10] and Wieber [11] for example adjusted the CoM dynamics to maintain balance; Herdt et al. [5] isolated the discrete aspect of changes in the BoS in a priorly-defined activation matrix, allowing to add the determination of the footsteps location to the MPC problem.

However, the time of activation of discrete changes in the BoS might noticeably affect the performance of both balance and the walking task. This section therefore describes a model considering simultaneously the CoM dynamics, amplitude and instants of changes in the BoS. Aiming at a computationally-efficient formulation, the use of a redundant set of integer and real variables to describe the contact state of the robot allows to keep a linear form of the various constraints the system is subject to.

\section{A. Mixed-Integer biped model}

To capture the discrete nature of changes in the BoS, with a view to future optimization, a choice of linearlyconstrained, redundant descriptors of the contact state is proposed. Additional details can be found in [12].

The amplitude and position of the $\mathrm{BoS}$ are described by its bounding box, as illustrated in Figure 2(a): real-valued variables $(\boldsymbol{a}, \boldsymbol{b}) \in \mathbb{R}^{2} \times \mathbb{R}^{2}$ are defined as the upper and lower bounds, respectively, of the position in the two horizontal directions of the feet in contact. The discrete essence of changes in the BoS leads to the definition of several con- 
straints, called shape constraints, restraining variables $(\boldsymbol{a}, \boldsymbol{b})$. Essentially, $(\boldsymbol{a}, \boldsymbol{b})$ must be piecewise-constant. This constraint can be linearly expressed with the introduction of binary variables $(\boldsymbol{\alpha}, \boldsymbol{\beta}) \in\{0,1\}^{2} \times\{0,1\}^{2}$ as rising and falling edges of $\boldsymbol{a}$ and $\boldsymbol{b}$, respectively.

Bounds $(\boldsymbol{a}, \boldsymbol{b})$ are furthermore implicitly related to feet positions: thus there exists couplings between the two directions of $\boldsymbol{a}$ and $\boldsymbol{b}$, called admissibility constraints.

First, single support phases (SS) impose that $\boldsymbol{a}$ equals $\boldsymbol{b}$, as the $\mathrm{BoS}$ is reduced to one foot ( $c f$. Figure 2(a)). SS phases must hence be differentiated from double support ones (DS): the binary variable $\gamma \in\{0,1\}$ is introduced to this purpose, and allows to define this constraint linearly.

Second, each change in the pair $(\boldsymbol{a}, \boldsymbol{b})$ leads to the alternation of $\gamma$, as any shift in the $\operatorname{BoS}$ corresponds to either a transition $\mathrm{SS} \rightarrow \mathrm{DS}$ or $\mathrm{DS} \rightarrow \mathrm{SS}$. Note that, as a result, $\gamma$ is fully defined by $(\boldsymbol{a}, \boldsymbol{b})$ and $(\boldsymbol{\alpha}, \boldsymbol{\beta})$ assuming that an initial value of $\gamma$ is known.

Last, potential changes in $\boldsymbol{a}$ and $\boldsymbol{b}$ from DS to SS depend on the configuration of the feet, relatively to bounds $\boldsymbol{a}$ and $\boldsymbol{b}$, in the previous DS phase. Indeed, as illustrated in Figure 2(a), only the pairs $\left(a_{1}, a_{0}\right)$ or $\left(b_{1}, b_{0}\right)$ can change when leaving the first DS configuration. On the contrary, leaving the last DS configuration can only lead to changes in the pairs $\left(b_{1}, a_{0}\right)$ or $\left(a_{1}, b_{0}\right)$. This coupling can be linearly expressed with the introduction of the binary variable $\delta \in\{0,1\}$ : the first configuration in Figure 2(a) corresponds to $\delta=1$ and the last to $\delta=0$. Note that this relation is bilateral: when leaving SS to DS, changes in $(\boldsymbol{a}, \boldsymbol{b})$ set the value of $\delta$, and when switching back to SS, the value of $\delta$ restrains potential changes in $(\boldsymbol{a}, \boldsymbol{b})$.

Figure 2(b) proposes typical evolutions of this set of BoS variables.

Contact state of the robot can therefore be described by the linearly-constrained, mixed-integer set of variables $(\boldsymbol{a}, \boldsymbol{b}, \boldsymbol{\alpha}, \boldsymbol{\beta}, \delta, \gamma)$. The ZMP model, neglecting rotational effects, relates CoM dynamics to the BoS; state $\boldsymbol{\xi}$ of the system in a balance perspective can thus be reduced to

$$
\boldsymbol{\xi}=\left[\begin{array}{llllllllll}
\boldsymbol{a} & \boldsymbol{b} & \boldsymbol{\alpha} & \boldsymbol{\beta} & \delta & \gamma & \boldsymbol{c} & \dot{\boldsymbol{c}} & \ddot{\boldsymbol{c}} & ]^{T},
\end{array}\right.
$$

where $c \in \mathbb{R}^{3}$ is the position of the CoM in the world frame. In the rest of this paper, time is sampled at discrete control instants $t_{i}$, and notation $v_{j}$ for function $v$ of time $t$ denotes the value $v\left(t_{j}\right)$, and $v_{k \mid j}$ the value $v\left(t_{j}\right)$ estimated from control time $t_{k}$. As stated earlier, the major contribution of such a description of the contact state is that, in discrete time, shape and admissibility constraints can be put in the linear form

$$
\forall k \in \mathbb{N}, \quad \mathbf{A}_{c l} \boldsymbol{\xi}_{k}+\mathbf{A}_{c r} \boldsymbol{\xi}_{k+1} \preceq \boldsymbol{f}_{c}
$$

where $\mathbf{A}_{c l}$ and $\mathbf{A}_{c r}$ are $n_{c} \times 19$ matrices, and $\boldsymbol{f}_{c}$ a vector in $\mathbb{R}^{n_{c}}$.

Biped postural stability, in non-sliding cases, can be obtained by avoiding tip-over of the humanoid: with coplanar contacts, the center of pressure (CoP) must stay away from the edges of the BoS. The ZMP approach [9] allows the computation of the position $\boldsymbol{p}$ on the ground of the CoP from the dynamics of the system. Ignoring rotational effects compared to translational ones, and neglecting vertical acceleration of the CoM compared to its horizontal acceleration, $\boldsymbol{p}$ writes

$$
\boldsymbol{p}=\boldsymbol{h}-\frac{\boldsymbol{c} \cdot \boldsymbol{e}_{2}}{g} \ddot{\boldsymbol{h}} \quad \text { where } \quad \boldsymbol{h}=\boldsymbol{c}-\left(\boldsymbol{c} \cdot \boldsymbol{e}_{2}\right) \boldsymbol{e}_{2},
$$

with $\boldsymbol{e}_{2}$ the ascendant vertical direction, $g$ the gravity amplitude and $\boldsymbol{h}$ the horizontal position of the CoM. In order to have a linear expression of $\boldsymbol{p}$ with respect to $\boldsymbol{\xi}$ and considering the approximation on the vertical acceleration of the CoM, the altitude of the CoM is considered as constant in the rest of this paper. The relevant CoM dynamics are thus the horizontal ones $\boldsymbol{h}$, therefore system state $\boldsymbol{\xi}$, matrices $\mathbf{A}_{c l}$ and $\mathbf{A}_{c r}$ and vector $\boldsymbol{f}_{c}$ are modified accordingly. Also, the balance constraint is linear with respect to the horizontal dynamics of the CoM, but changes in the supporting surface being reflected by changes in state variables $(\boldsymbol{a}, \boldsymbol{b})$, the inclusion constraint is quadratic with respect to the state $\boldsymbol{\xi}$ of the system. Nevertheless, as shown in [5], overestimating the convex hull of this surface by its bounding box in forward and lateral directions brings the definition of a set of linear inequality constraints with respect to $\boldsymbol{\xi}$. Note that this approximation is solely made for future potential DS phases: constraints for an established BoS can be fully implemented without overestimation ( $c f$. Figure 2(a)). Remarks on this approximation are provided in Section IV-D. Under this overestimation, the CoP constraints write

$$
\forall k \in \mathbb{N}, \quad \mathbf{A}_{p} \boldsymbol{\xi}_{k} \preceq \boldsymbol{f}_{p},
$$

where $\mathbf{A}_{p}$ is a $n_{p} \times 16$ matrix, and $\boldsymbol{f}_{p}$ a vector in $\mathbb{R}^{n_{p}}$.

The previous description of the contact state, with the ZMP model, brings the resulting mixed-integer biped model at time $t_{k}$

$$
\begin{aligned}
& \boldsymbol{\xi}_{k}=\left[\begin{array}{lllllllll}
\boldsymbol{a}_{k} & \boldsymbol{b}_{k} & \boldsymbol{\alpha}_{k} & \boldsymbol{\beta}_{k} & \delta_{k} & \gamma_{k} & \boldsymbol{h}_{k} & \dot{\boldsymbol{h}}_{k} & \ddot{\boldsymbol{h}}_{k}
\end{array}\right]^{T} \\
& \text { s.t. }\left\{\begin{aligned}
\mathbf{A}_{c, r} \boldsymbol{\xi}_{k} & \preceq \boldsymbol{f}_{c}-\mathbf{A}_{c, l} \boldsymbol{\xi}_{k-1}, \\
\mathbf{A}_{p} \boldsymbol{\xi}_{k} & \preceq \boldsymbol{f}_{p}, \\
\left(\boldsymbol{\alpha}_{k}, \boldsymbol{\beta}_{k}\right) & \in\{0,1\}^{2} \times\{0,1\}^{2}, \\
\left(\delta_{k}, \gamma_{k}\right) & \in\{0,1\} \times\{0,1\}, \\
\left(\boldsymbol{a}_{k}, \boldsymbol{b}_{k}\right) & \in \mathbb{R}^{2} \times \mathbb{R}^{2}, \\
\left(\boldsymbol{h}_{k}, \dot{\boldsymbol{h}}_{k}, \ddot{\boldsymbol{h}}_{k}\right) & \in \mathbb{R}^{2} \times \mathbb{R}^{2} \times \mathbb{R}^{2},
\end{aligned}\right.
\end{aligned}
$$

which describes and restrains the balance state of the system.

\section{B. Walking motion constraints}

The model described in (5) allows the consideration of a large variety of linear constraints, inherent to the control problem of walking humanoids. Relevant constraints regarded in this paper are maximum leg span, maximum swinging foot average velocity and minimal SS/DS phases durations. Such constraints will ban strategies involving too large or fast steps, and hinder solutions such as fast series of small steps. Note that additional constraints such as maximal SS/DS durations, bounds on the CoM velocity and 
acceleration, or bounds on positions of the feet can also be put in a linear form with respect to the system state $\boldsymbol{\xi}$.

Let $\bar{v}$ denote the maximal swinging foot velocity, $\bar{s}$ the maximal step length and $\bar{t}$ the largest of minimum durations of SS and DS phases. Constraints on leg span, foot velocity and SS/DS durations write

$$
\sum_{t_{k+j}-t_{k} \leq \max (\bar{s} / \bar{v}, \bar{t})} \mathbf{A}_{w, j} \boldsymbol{\xi}_{k+j} \preceq \boldsymbol{f}_{w}, \quad j \in \mathbb{N},
$$

where $\mathbf{A}_{w, j}$ is a $n_{w} \times 19$ matrix, and $\boldsymbol{f}_{w}$ a vector in $\mathbb{R}^{n_{w}}$. It can be shown that $\boldsymbol{f}_{w}$ depends on a history of states $\boldsymbol{\xi}$ prior to $t_{k}$, and can be written in the form

$$
\boldsymbol{f}_{w}=\boldsymbol{f}_{w_{0}}+\sum_{t_{k}-t_{k-j}<\max (\bar{s} / \bar{v}, \bar{t})} \mathbf{B}_{w, j} \boldsymbol{\xi}_{k-j}, \quad j \in \mathbb{N}^{*} .
$$

Condition $t_{k+j}-t_{k} \leq \max (\bar{s} / \bar{v}, \bar{t})$ (resp. $t_{k}-t_{k-j}$ ) states that the forward (resp. backward) history influence is irrelevant beyond the time step where both minimum duration and maximum average velocity constraints are necessarily respected. A constraint to avoid the overlapping of feet is added, in the form of a linearized collision avoidance constraint.

This model is employed in the following section to evaluate and optimize the performance, in terms of walking activity and balance, of admissible evolutions of the system state.

\section{QUADRATIC, MIXED-INTEGER MPC}

The walking control problem, in a predictive framework, consists in finding an optimal and admissible horizon of future system states $\boldsymbol{\xi}_{k \mid k+j}$ that ensure the performance of an ongoing walking activity while maximizing balance of the system. The descriptors of the system state presented in Section II being taken as variables of an optimization problem, these objectives can be written in the form of quadratic functions, leading to the formulation of a MPC problem as a mixed-integer quadratic program.

Considering approximations exploited in the ZMP model, a minimal set of parameters can be obtained to describe an horizon of system states $\boldsymbol{\xi}_{k \mid k+j}$. Indeed, a discrete integration scheme brings, with piecewise constant CoM jerks $\boldsymbol{u}=$ $\partial^{3} \boldsymbol{h} / \partial t^{3}$

$$
\forall j \in \mathbb{N}^{*}, \quad \hat{\boldsymbol{h}}_{k \mid k+j+1}=\mathbf{A}_{h} \hat{\boldsymbol{h}}_{k \mid k+j}+\mathbf{B}_{h} \boldsymbol{u}_{k \mid k+j+1},
$$

where $\hat{\boldsymbol{h}}=\left[\begin{array}{lll}\boldsymbol{h} & \boldsymbol{h} & \ddot{\boldsymbol{h}}\end{array}\right]^{T}$ is the CoM horizontal dynamics, and $\mathbf{A}_{h}, \mathbf{B}_{h}$ integration matrices. The minimal set of variables required to preview $\boldsymbol{\xi}_{k \mid k+j+1}$ from $\boldsymbol{\xi}_{k \mid k+j}$ is hence denoted $\chi_{k \mid k+j+1}$, defined as

$$
\chi=\left[\begin{array}{llllllll}
\boldsymbol{a} & \boldsymbol{b} & \boldsymbol{\alpha} & \boldsymbol{\beta} & \delta & \gamma & \boldsymbol{u}
\end{array}\right]^{T} .
$$

The model (5) can thus be written as the Linear TimeInvariant process

$$
\forall j \in \mathbb{N}^{*}, \quad \boldsymbol{\xi}_{k \mid k+j+1}=\mathbf{Q} \boldsymbol{\xi}_{k \mid k+j}+\mathbf{T} \boldsymbol{\chi}_{k \mid k+j+1},
$$

where $\mathbf{Q}$ and $\mathbf{T}$ are state description matrices derived from (8). Relation (9) allows to preview an horizon $\boldsymbol{C}_{k, N}$ of $N$ future states $\boldsymbol{\xi}_{k \mid k+j}$ from an horizon $\boldsymbol{X}_{k, N}$ of $N$ future inputs $\chi_{k \mid k+j}$ and the actual state $\boldsymbol{\xi}_{k \mid k}=\boldsymbol{\xi}_{k}$. The preview writes, with $\mathbf{P}$ and $\mathbf{R}$ combinations of $\mathbf{Q}$ and $\mathbf{T}$

$$
\boldsymbol{C}_{k, N}=\left[\begin{array}{c}
\boldsymbol{\xi}_{k \mid k+1} \\
\vdots \\
\boldsymbol{\xi}_{k \mid k+N}
\end{array}\right]=\mathbf{P} \boldsymbol{\xi}_{k}+\mathbf{R}\left[\begin{array}{c}
\chi_{k \mid k+1} \\
\vdots \\
\boldsymbol{\chi}_{k \mid k+N}
\end{array}\right]
$$

denoted as

$$
\boldsymbol{C}_{k, N}=\mathbf{P} \boldsymbol{\xi}_{k}+\mathbf{R} \boldsymbol{X}_{k, N} .
$$

Linear equation (10) allows to reformulate the linear equalities (2), (4) and (6) as

$$
\mathbf{A} \boldsymbol{X}_{k, N} \preceq \boldsymbol{f}
$$

where $\boldsymbol{f}$ depends on a history of actual and previous states $\boldsymbol{\xi}$, as shown in (2), (6) and (7).

A walking activity can be interpreted as reaching a target position with a desired horizontal velocity, which can be expressed at the level of the CoM. It is in such terms a tracking task, whose performance can be evaluated as a tracking error. Let $\hat{\boldsymbol{h}}^{\text {ref }}$ denote the desired trajectory of the CoM. Objective $J_{w}$ of the walking activity can be written, over a preview horizon, as the minimization of

$$
J_{w k}=\sum_{j=1}^{N}\left\|\mathbf{S}\left(\hat{\boldsymbol{h}}_{k \mid k+j}-\hat{\boldsymbol{h}}_{k \mid k+j}^{r e f}\right)\right\|^{2},
$$

where $\mathbf{S}$ is a $6 \times 6$ weighting selection matrix, diagonal, defining whether position, velocity and/or acceleration are tracked in each of the two horizontal directions. For example, a standstill activity aiming at $\dot{\boldsymbol{h}} \rightarrow \mathbf{0}$ can be expressed with $\hat{\boldsymbol{h}}^{r e f}=\mathbf{0}$ and the only non-null terms of $\mathbf{S}$ corresponding to velocity in both horizontal directions; that is, $J_{w k}=$ $\sum_{j=1}^{N}\left\|\dot{\boldsymbol{h}}_{k \mid k+j}\right\|^{2}$.

While performance of the walking activity is essential, robustness of the posture of the robot is also a major objective in walking motions. Balance is guaranteed, under the assumptions of the model, by the CoP constraint (4) in (11). Nevertheless, as a tip-over situation occurs when the $\mathrm{CoP}$ reaches the edges of the $\mathrm{BoS}$, robustness of the balance state of the robot can be captured as a distance to the edges. The balance maximization objective can thus be written as the minimization of $J_{b}$

$$
J_{b k}=\sum_{j=1}^{N}\left\|\boldsymbol{p}_{k \mid k+j}-\boldsymbol{r}_{k \mid k+j}\right\|^{2},
$$

where $\boldsymbol{r}=(\boldsymbol{a}+\boldsymbol{b}) / 2$ is the center of the BoS, i.e. the point at the greatest distance from the edges of the supporting surface.

Secondary objectives are added to the control problem in these works for regularization purposes. They aim at minimizing CoM jerks, avoid excessive changes in solutions from one control step to another, keep track of the previous BoS 
size and prefer DS phases over SS ones. These regularization objectives are written in the form of a quadratic cost $q$, which can be used to reward desired behaviors in order to favor short and frequent stepping over slower gaits, for example.

Objectives (12) and (13) are quadratic with respect to $\boldsymbol{X}_{k, N}$, and are considered in the global cost function $J$

$$
J_{k}=\omega_{b} J_{b k}+\omega_{w} J_{w k}+q=\boldsymbol{X}_{k, N}^{T} \mathbf{H} \boldsymbol{X}_{k, N}+\boldsymbol{d}^{T} \boldsymbol{X}_{k, N},
$$

where $\mathbf{H}$ is a positive definite matrix, $\boldsymbol{d}$ a vector and $\left(\omega_{b}, \omega_{w}\right)$ scalar weights defining a compromise between balance robustness and tracking performance. The MPC problem finally writes

$$
\text { s.t. }\left\{\begin{aligned}
& \min _{\boldsymbol{X}_{k, N}} \boldsymbol{X}_{k, N}^{T} \mathbf{H} \boldsymbol{X}_{k, N}+\boldsymbol{d}^{T} \boldsymbol{X}_{k, N} \\
& \mathbf{A} \boldsymbol{X}_{k, N} \preceq \boldsymbol{f} \\
& \boldsymbol{\xi}_{k \mid k}=\boldsymbol{\xi}_{k} \\
& \boldsymbol{\xi}_{k \mid k+j+1}=\mathbf{Q} \boldsymbol{\xi}_{k \mid k+j}+\mathbf{T} \boldsymbol{\chi}_{k \mid k+j+1}, \\
&\left(\boldsymbol{a}_{k \mid k+j}, \boldsymbol{b}_{k \mid k+j}\right) \in \mathbb{R}^{2} \times \mathbb{R}^{2}, \\
&\left(\boldsymbol{\alpha}_{k \mid k+j}, \boldsymbol{\beta}_{k \mid k+j}\right) \in\{0,1\}^{2} \times\{0,1\}^{2}, \\
&\left(\delta_{k \mid k+j}, \gamma_{k \mid k+j}\right) \in\{0,1\} \times\{0,1\}, \\
& \boldsymbol{u}_{k \mid k+j} \in \mathbb{R}^{2},
\end{aligned}\right.
$$

which is a Mixed-Integer Quadratic Program in canonical form. The computational complexity of problem (15) is strongly related to the number of non-real variables. Nevertheless its QP form allows the use of fast algorithms and binary variables can be sampled at a lower frequency, as discussed in Section IV-D.

\section{RESULTS}

Contribution of the MPC formulation (15) is exposed with several simulation results, demonstrating the variety of behaviors generated in diverse scenarii.

Opening results illustrate that, without the use of any heuristics other than motion constraints and controller weights, the MPC (15) automatically generates an intuitive gait pattern to follow a varying reference velocity. A second set of results exhibits optimal modifications of the gait pattern to accommodate an ongoing walking activity to large physical disturbances on the robot. Last, results in a pushrecovery scenario demonstrate how weights in (14) allows to implicitly define, at a high level, different behaviors of the humanoid.

Simulations are performed using the ARBORIS-PYTHON simulator [13] developed at ISIR, and whole-body motion from optimal outputs of the MPC (15) is ensured by an LQP-based controller [14] for an iCub [15] robot model. The system state is previewed over an horizon of $1.0 \mathrm{~s}$, and simulations are run with a time step of $d t=1 \cdot e^{-2} s$. The humanoid weighs $\approx 27 \mathrm{~kg}$ with an height of $\approx 1 \mathrm{~m}$.

\section{A. Gait generation: sinusoidal velocity tracking}

A first simulation scenario is performed to illustrate the behavior of the MIQP problem in nominal conditions. A walking activity is specified as the tracking of a sinusoidal CoM velocity in the forward direction, and a null velocity in the lateral direction. Major weights $\left(\omega_{b}, \omega_{w}\right)$ in the cost

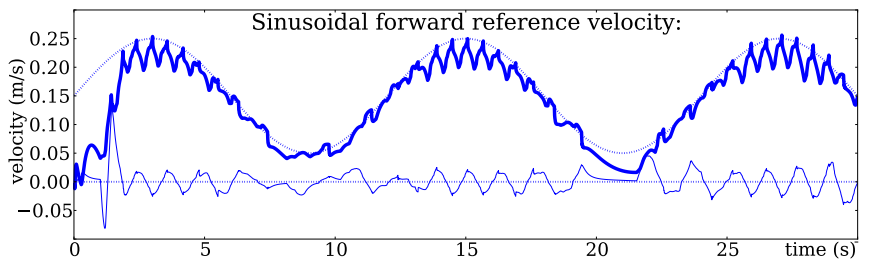

Fig. 3. CoM velocity tracking performance - thick line: forward thin line: lateral — dotted lines: reference velocities

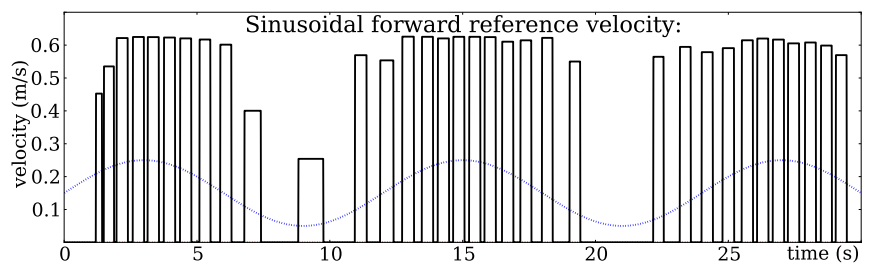

Fig. 4. Generated gait pattern from CoM velocity tracking — solid: average foot velocity during single support-dotted: CoM reference velocities

function (14) are equal, and forward and lateral directions have the same importance in the tracking error function (12), i.e. $S_{i j} \in\{0,1\}$.

Performance of the tracking task is depicted in Figure 3: both forward and lateral CoM velocity tend towards the reference $\dot{\boldsymbol{h}}^{\text {ref }}$. However, oscillations are still visible as the result of the alternation between SS and DS phases. Indeed, velocity of the CoM is restrained by the CoP constraints (4) its acceleration is subject to, constraints that are all the more restrictive in single support phases. Consequently, velocity tracking is hindered during SS phases, and DS phases release DoFs of the CoM, allowing the recovery of the tracking task. Nevertheless, in periods of fast reference velocity $\dot{h}^{\text {ref }}$, a new SS phase is rapidly required and the robot do not have time, due to regularizing terms $q$, to fully reach the required velocity.

This last effect is illustrated in Figure 4 which displays SS/DS alternations with the average foot velocity during each SS phase. Indeed, time periods of high reference velocity such as $t=15 \mathrm{~s}$ require steps of high velocity and frequency, while periods of medium reference velocity such as $t \in$ $[10 s, 13 s]$ lead to slower, sparser steps and a better CoM velocity tracking.

From a more distant point of view, Figure 4 exposes an intuitive strategy computed from (15): steps taken automatically tend to be longer, faster and more frequent as the desired walking velocity grows higher.

Note that equal weights $\omega_{b}=\omega_{w}$ in (14) tend to give a

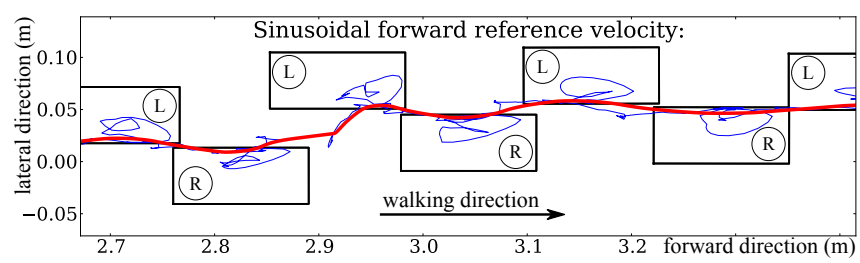

Fig. 5. Evolution of the actual $\mathrm{CoP}$ and $\mathrm{CoM}$ with generated footsteps — thick line: CoM position - thin line: actual CoP position 

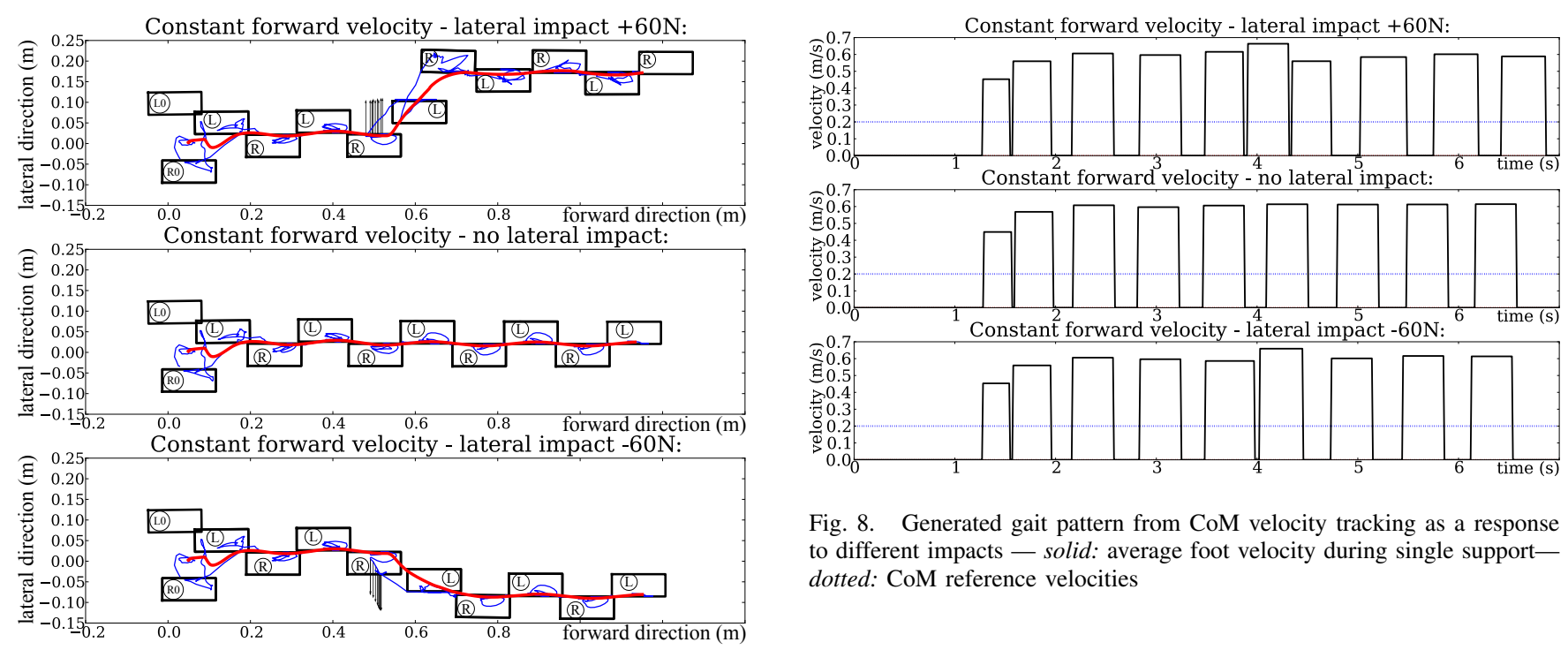

Fig. 8. Generated gait pattern from CoM velocity tracking as a response to different impacts - solid: average foot velocity during single supportdotted: CoM reference velocities

Fig. 7. Evolution of the actual $\mathrm{CoP}$ and $\mathrm{CoM}$ with the adaptation of generated footsteps to an unknown impact - thick line: CoM position thin line: actual $\mathrm{CoP}$ position

higher priority to the walking task, particularly in cases of high velocity tracking. This is mainly due to objectives $J_{w}$ and $J_{b}$ not being normalized, hence letting differences in magnitude have an influence. This effect can be observed in Figure 5 which presents an extract of $\mathrm{CoM}, \mathrm{CoP}$ and feet positions. Although balance is still ensured, the tracking task slightly prevents, especially in SS phases, the CoP from staying in the middle of the BoS.

Figure 5 shows that the feet tend to be aligned in the forward direction as a result of the null velocity tracking in the lateral direction, in order to minimize the lateral velocity of the CoM during footsteps. However, cases of low forward reference velocities require small steps: the non-overlapping constraint on the feet forces a lateral gap between them, hence inducing a loss of performance of the tracking task in the lateral direction, as shown in Figure 3 around $t=22 \mathrm{~s}$ for example.

\section{B. Activity adjustments: walking under large disturbances}

This simulation aims at exhibiting, for an identical set of weights in the cost function (14), differences in the optimal behavior against unknown disturbances. The humanoid has to perform a walking task with a target forward velocity of $0.20 \mathrm{~m} \cdot \mathrm{s}^{-1}$, and a null desired lateral velocity. Three cases are compared: an unknown lateral impact of $+60 N$ (more thant a fifth of the total weight of the robot) is applied to the head of the humanoid during a period of $0.1 s$, then no effort is applied to the robot and, last, an opposite impact of $-60 \mathrm{~N}$ is considered. Note that applying the external action to the head brings additional disturbances as large rotational effects are induced, effects which are not taken into account in the ZMP model (3). Snapshots of the different cases are provided in Figure 6.

Figure 7 shows that changes in the system state from the impact lead to an intuitive modification of the steps taken:

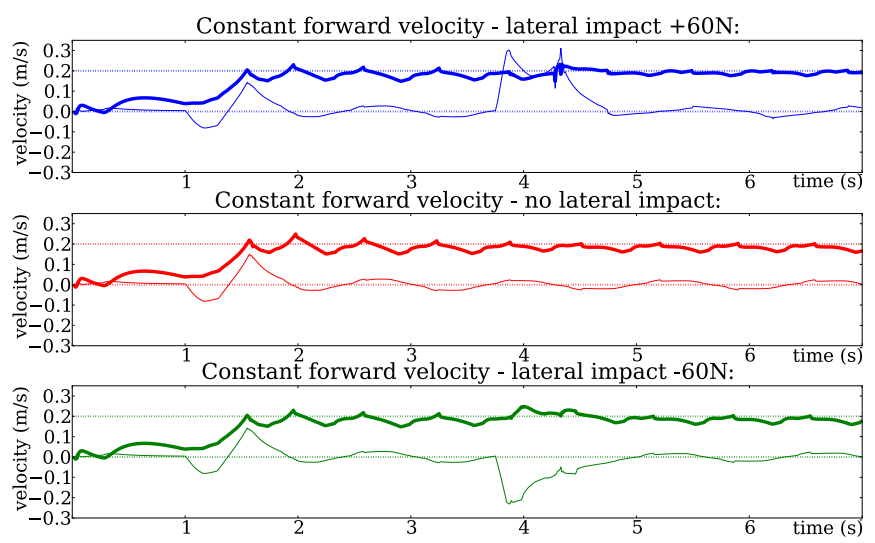

Fig. 9. CoM velocity tracking performance against the impact thick line: forward - thin line: lateral — dotted lines: reference velocities

balance is ensured with a lateral drift of the robot in the direction of the impact. It can be noted that for the impact of $+60 N$ the right foot crosses the left one: such a solution can be excluded by a set of linear constraints in (15), or selfcollisions should be handled at the whole-body control level. The impact occurring during an ongoing step, Figure 8 shows that the speed and duration of this step is slightly altered as a response to the disturbance. Moreover, a major aspect of the recovery strategy is illustrated: the DS phase following the impact is noticeably shorter than in the undisturbed case. A lateral step is indeed taken almost immediately in order to improve balance.

These modifications in the gait pattern as a response to the impact seem natural from the balance point of view. Nevertheless, as illustrated in Figure 9, although a null lateral velocity obviously cannot be reached during the impact, the desired CoM velocities are rapidly recovered. Also, it can be noted that the tracking task in the forward direction is almost unaffected by the impact. The recovery steps taken are hence compatible with the ongoing walking activity, as objective function $J_{w}$ is still part of the optimization problem. 


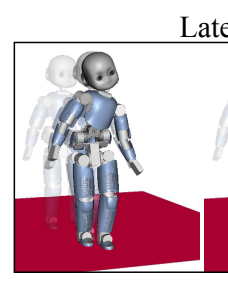

Lateral impact $60 \mathrm{~N}$
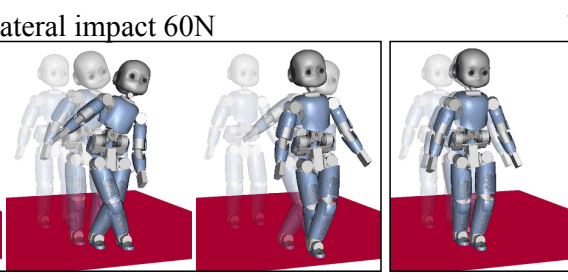

No lateral impact
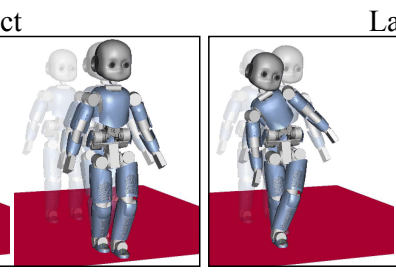

Lateral impact $-60 \mathrm{~N}$
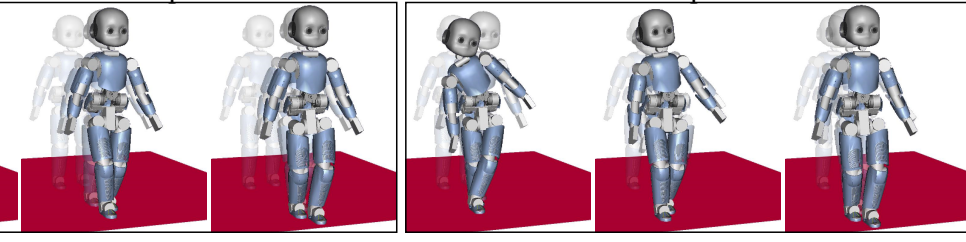

Fig. 6. Snapshots of a walking activity under different unknown external impacts, applied to the head of the humanoid

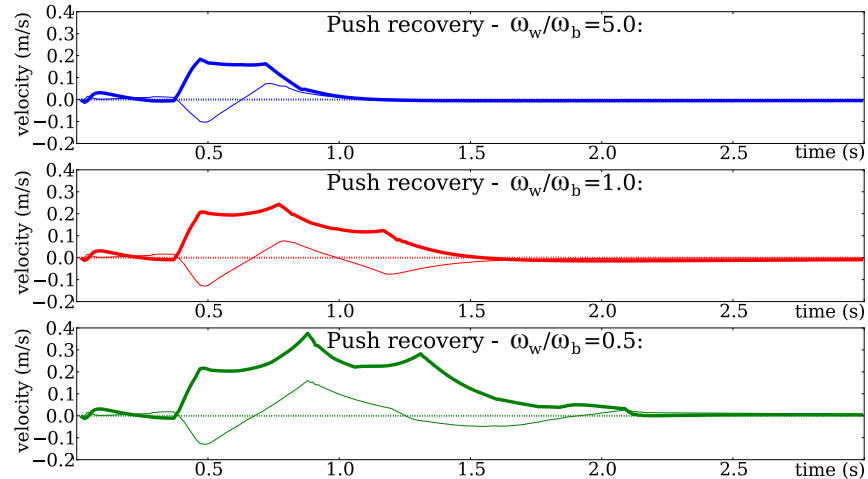

Fig. 10. Influence of the objective weights on the CoM velocity tracking performance - thick line: forward - thin line: lateral — dotted lines: reference velocities

\section{Behavior specification: push-recovery}

A last simulation scenario demonstrates how the balance behavior of the humanoid can be specified with the sole ratio $\omega_{w} / \omega_{b}$. Differences in behavior are illustrated in a push recovery case. The humanoid must stand still, i.e. $\dot{\boldsymbol{h}}^{\text {ref }}=\mathbf{0}$, and experiences an unknown impact of $50 \mathrm{~N}$ in the forward direction and $-25 N$ in the lateral one, during a period of $0.1 s$. The standing task is incrementally relaxed in three cases. A first set of weights targets the best activity performance with $\omega_{w} / \omega_{b}=5.0$, a second is placed as reference with $\omega_{w} / \omega_{b}=1.0$ and a last set gives a higher priority to the balance objective $J_{b}$ with $\omega_{w} / \omega_{b}=0.5$. The difference in the resulting behavior of the humanoid is illustrated in figures 10, 11 and 12 for the three cases.

As expected, results provided in Figure 10 show that priority of the tracking objective $J_{w}$, relatively to $J_{b}$, has a direct influence on the evolution of the CoM velocity. Although the humanoid eventually reaches the null reference velocity in all cases, control with $\omega_{w} / \omega_{b}=5.0$ attains the standstill objective around three times faster than with $\omega_{w} / \omega_{b}=0.5$.

This gain in performance is achieved with a noticeably different gait pattern for the three cases. Indeed, as shown in Figure 11 the first controller stops the CoM with one step, the second with two and the third recovers a standstill state after three steps. It can be observed that the duration of the first recovery step grows with the decrease of the ratio $\omega_{w} / \omega_{b}$, which can be interpreted as the concurrence of two effects. During SS phases, the dynamics of the CoM are conditioned by the $\mathrm{CoP}$ constraint (4), hence potentially affecting the tracking objective $J_{w}$ as shown in Figure 10; on the other hand, a longer step duration allows to place

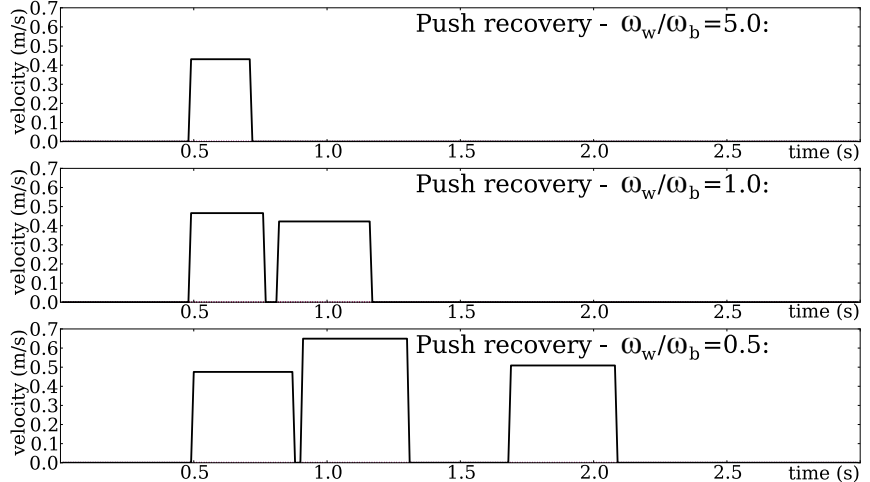

Fig. 11. Generated gait pattern from CoM velocity tracking for different objective weights in a push recovery scenario — solid: average foot velocity during single support

the BoS as desired, in favor of the balance objective $J_{b}$. Essentially, DS phases provides the CoM with a greater degree of freedom, while SS phases are required to adapt the $\mathrm{BoS}$ in regard to the balance objective.

Figure 12 indeed confirms these remarks. The first recovery step tends to be further away from the initial feet configuration as the ratio $\omega_{w} / \omega_{b}$ decreases, which leads, in average, to a better placement of the CoP with respect to the BoS.

\section{Remarks and discussions}

As observed in figures 5, 7 and 12, the CoP happens to reach the edges of the BoS, thus putting the system at tipover risk although constraints on the CoP (4) are specified with a safety margin of $\approx 30 \%$, and are guaranteed in problem (15). The ZMP model (3) used as an approximation of the CoP ignores, among others, rotational effects. However such effects may yet be of large magnitude in the cases studied in this section: fast reference velocities and sudden disturbances are considered, leading to rapid steps and, as a consequence, fast movements of the swinging leg. Moreover, the application of the external force to the head of the robot induces rotational effects from the entire upper body of the humanoid ( $c f$. figure 6), thus increasing the gap between the actual CoP and estimated ZMP (3). Note that this gap might be partially reduced by setting a lower maximal foot velocity. Another approximation regarding the $\mathrm{CoP}$ is the overestimation of the convex hull of the contact points by its bounding box, in order to write linear constraints with respect to state $\boldsymbol{\xi}$. Nevertheless, outputs from (15) can be validated at each control step to ensure that the previewed $\mathrm{CoP}$ remains inside the convex hull of the BoS. If not, a fast QP program 

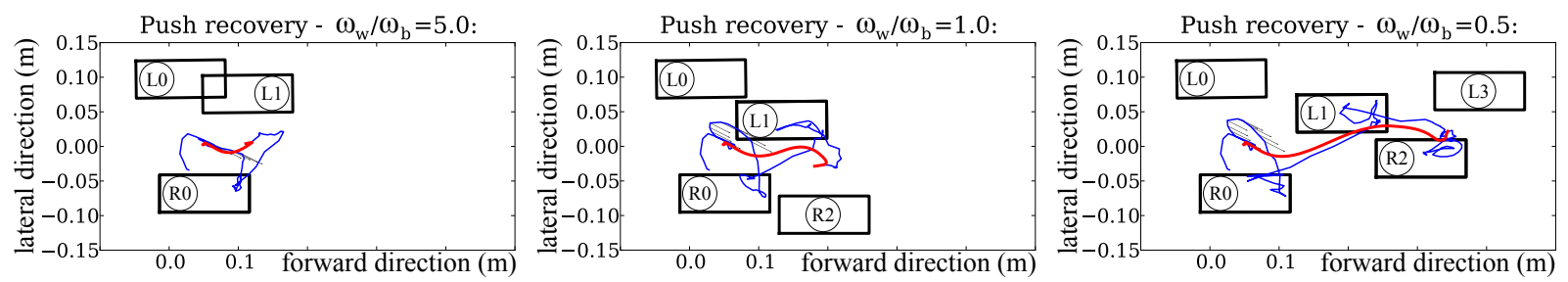

Fig. 12. Evolution of the actual CoP and CoM with generated footsteps for an identical impact and different objective weights $\left(\omega_{b}, \omega_{w}\right)-$ thick line: CoM position - thin line: actual CoP position

as described in [11] can be set up to recompute a valid CoM trajectory (possibly with new feet positions as in [5]), taking as input the changes in BoS provided by (15). Nevertheless, such a safety setup was not necessary in the presented simulations.

Results introduced in this section were obtained with the GUROBi Optimizer [16]. A non uniform time sampling scale was used, in order to keep a reduced size of the optimization vector while avoiding the accumulation of integration errors in the preview. Furthermore, variables associated to changes in the BoS were sampled at a lower frequency than variables describing the CoM dynamics. Indeed, the complexity of the MIQP (15) is strongly related to the number of integer variables, and it can arbitrarily be considered that changes in the $\mathrm{BoS}$ occur at a lower frequency than CoM adjustments. Solving the MIQP (15) took an average of $40 \mathrm{~ms}^{1}$, and note that since MIQP solvers generally employ branch-and-bound based algorithms, computation time can largely be influenced by the order of variables and the use of heuristics, helping at reaching the best nodes faster.

Lastly, it can be noted that the model of the BoS presented in Section II omits DoFs from the rotations of the feet. Nevertheless, orienting the BoS in the motion or disturbance direction may be beneficial to balance.

\section{CONCLUSIONS}

A novel predictive formulation of the walking control problem is validated in this paper. The consideration of all admissible changes in the BoS adds DoFs to the behavior of the system, behavior which can be specified with a highlevel lever in the form of a weight ratio between balance and walking. Simulation results show a large range of balance strategies adopted by the humanoid, independently from prior gait patterns and moderated by the imposed compromise between balance and walking performance, as a response to the ongoing activity and unknown disturbances on the system.

Future works may consider rotations of the feet to generate a wider range of motions, and investigate in further details the computational efficiency of the problem. Additional

\footnotetext{
${ }^{1}$ on a dual-core i7-2620M for ?? DoFs and ?? constraints. The computation time being greater than the timestep, simulation was slowed down in this work. Future works will investigate the implementation of a slower control loop for gait adaptation.
}

developments may also consider configurations with noncoplanar contacts.

\section{ACKNOWLEDGMENT}

This work was partially supported by the French Ministry of Higher Education and Research, by the RTE company through its chair "Robotics Systems for field intervention in constrained environments" hold by Vincent Padois and by the European Commission within the CoDyCo project (FP7ICT-2011-9, No. 600716).

\section{REFERENCES}

[1] A. F. Miguel, "The emergence of design in pedestrian dynamics: Locomotion, self-organization, walking paths and constructal law," Physics of life reviews, 2013.

[2] V. Prahlad, G. Dip, and C. Meng-Hwee, "Disturbance rejection by online zmp compensation," Robotica, vol. 26, no. 1, p. 9, 2008.

[3] S. Kanzaki, K. Okada, and M. Inaba, "Bracing behavior in humanoid through preview control of impact disturbance," in Proc. of the IEEERAS Int. Conf. on Humanoid Rob. IEEE, 2005, pp. 301-305.

[4] J. Pratt, J. Carff, S. Drakunov, and A. Goswami, "Capture point: A step toward humanoid push recovery," in Proc. of the IEEE-RAS Int. Conf. on Humanoid Rob. IEEE, 2006, pp. 200-207.

[5] A. Herdt, H. Diedam, P.-B. Wieber, D. Dimitrov, K. Mombaur, and M. Diehl, "Online walking motion generation with automatic footstep placement," Advanced Robotics, vol. 24, no. 5-6, pp. 719-737, 2010.

[6] B. E. Maki, W. E. Mcilroy, and G. R. Fernie, "Change-in-support reactions for balance recovery," Engineering in Medicine and Biology Magazine, IEEE, vol. 22, no. 2, pp. 20-26, 2003.

[7] M. Krause, J. Englsberger, P.-B. Wieber, and C. Ott, "Stabilization of the capture point dynamics for bipedal walking based on model predictive control," in Robot Control, vol. 10, no. 1, 2012, pp. 165171.

[8] I. Mordatch, E. Todorov, and Z. Popović, "Discovery of complex behaviors through contact-invariant optimization," ACM Transactions on Graphics (TOG), vol. 31, no. 4, p. 43, 2012.

[9] M. Vukobratović and J. Stepanenko, "On the stability of anthropomorphic systems," in Mathematical Biosciences, vol. 15, no. 1, 1972, pp. $1-37$.

[10] S. Kajita, F. Kanehiro, K. Kaneko, K. Kajiwara, K. Harada, K. Yokoi, and $\mathrm{H}$. Hirukawa, "Biped walking pattern generation by using preview control of zero-moment point," in Proc. of the IEEE ICRA, 2003.

[11] P.-B. Wieber, "Trajectory free linear model predictive control for stable walking in the presence of strong perturbations," in Proc. of the IEEERAS Int. Conf. on Humanoid Rob., 2006, pp. 137-142.

[12] A. Ibanez, P. Bidaud, and V. Padois, "Automatic optimal biped walking as a mixed-integer quadratic program," in Submited to Advances in Robot Kinematics, J. Lenarcic and B. Roth, Eds. Springer, 2014.

[13] S. Barthelemy, J. Salini, and A. Micaelli, "Arboris-python.” [Online]. Available: https://github.com/salini/arboris-python

[14] J. Salini, V. Padois, and P. Bidaud, "Synthesis of complex humanoid whole-body behavior: a focus on sequencing and tasks transitions," in Proc. of the IEEE ICRA. IEEE, 2011, pp. 1283-1290.

[15] G. Sandini, G. Metta, and D. Vernon, "The icub cognitive humanoid robot: An open-system research platform for enactive cognition," in 50 Years of Artificial Intelligence, ser. Lecture Notes in Computer Science. Springer, 2007, ch. 32, pp. 358-369.

[16] I. Gurobi Optimization. [Online]. Available: http://www.gurobi.com 